\title{
Does the addition of induction chemotherapy improve outcome in locally advanced rectal carcinoma?
}

\author{
Francesca De Felice $^{1} \cdot$ Daniela Musio $^{1} \cdot$ Vincenzo Tombolini $^{1,2}$
}

Accepted: 13 June 2015 / Published online: 23 June 2015

(C) Springer-Verlag Berlin Heidelberg 2015

\section{Dear Editor:}

Locally advanced rectal cancer (LARC) is defined as directly tumor invasion into the pericolorectal tissues (T3) and other organs or structures (T4), and/or metastasis in regional lymph node $(\mathrm{N}+)$. The treatment of LARC is multidisciplinary, and neo-adjuvant chemoradiotherapy (CRT) followed by surgery is the standard of care in this setting of patients, based on the CAO/ARO/AIO-94 German Rectal Cancer Study. This study compared neo-adjuvant versus adjuvant CRT regimen in a cohort of 823 patients with LARC. Neo-adjuvant CRT improved local control ( 6 versus $13 \%, p=0.02$ ), as well as sphincter preservation (39 versus $19 \%, p=0.004$ ) and decreased acute and late toxicity ( 27 versus $40 \%$ and 14 versus $24 \%$, respectively) compared with post-operative CRT.

Year after year, local recurrence has represented the prevailing method of treatment failure. The degree of pathological regression, as well as the pathological complete response (pCR), correlates with a favorable prognosis. Nowadays, with the standardization of total mesorectal excision (TME) surgery and the advance in radiotherapeutic techniques, local tumor control rates have improved, whereas the development of distant metastasis remains the main mode of failure [1-4]. Thus, the reduction in local recurrence rates emphasizes the need to investigate the risk of distant metastases, and therefore the attention shifts to new treatment strategies to improve distant tumor control. Consequently, there is a rationale of adding

Francesca De Felice fradefelice@hotmail.it

1 Department of Radiotherapy, Policlinico Umberto I "Sapienza" University of Rome, Viale Regina Elena 326, 00161 Rome, Italy

2 Spencer-Lorillard Foundation, Viale Regina Elena 262, Rome, Italy induction chemotherapy (CHT): most of LARC recurrences are systemic and induction CHT should be used to better control distant metastasis.

Based on the efficacy demonstrated in stage IV colon cancer patients, the addition of induction chemotherapy should confirm beneficial properties. The theoretical advantage of induction CHT may allow improvement of oxygenation and higher intramural concentration of cytotoxic drugs. Benefit probably includes a tumor down staging and an early eradication of micrometastasis, without affect compliance on subsequent CRT and/or surgery.

The current aim is to evaluate the effect of induction CHT before neo-adjuvant CRT in LARC, to determine whether the induction CHT can assure better overall survival and disease free survival, which is typically achieved by concomitant neo-adjuvant treatment in patients with LARC.

\section{References}

1. Gérard JP, Conroy T, Bonnetain F, Bouché O, Chapet O, Clo-sonDejardin MT, Untereiner M, Leduc B, Francois E, Maurel J, Seitz JF, Buecher B, Mackiewicz R, Ducreux M, Bedenne L (2006) Preoperative radiotherapy with or without concurrent fluorouracil and leucovorin in T3-4 rectal cancers: results of FFCD 9203. J Clin Oncol 24:4620-4625

2. Havenga K, Grossmann I, DeRuiter M, Wiggers T (2007) Definition of total mesorectal excision, including the perineal phase: technical considerations. Dig Dis 25:44-50

3. Bolognese A, Cardi M, Muttillo IA, Barbarosos A, Bocchetti T, Valabrega S (2000) Total mesorectal excision for surgical treatment of rectal cancer. J Surg Oncol 74:21-23

4. Piso P, Dahlke MH, Mirena P, Schmidt U, Aselmann H, Schlitt HJ, Raab R, Klempnauer J (2004) Total mesorectal excision for middle and lower rectal cancer: a single institution experience with 337 consecutive patients. J Surg Oncol 86:115-121 